\title{
Indicadores epidemiológicos da hanseníase em Sergipe: Um olhar direcionado para os Determinantes Sociais da Saúde
}

\author{
Epidemiological indicators of leprosy in Sergipe: A look towards the Social Determinants of Health \\ Indicadores epidemiológicos de la lepra en Sergipe: Una mirada a los determinantes sociales de la \\ salud
}

\author{
Thayane Santos Siqueira \\ ORCID: https://orcid.org/0000-0001-8370-0544 \\ Universidade Federal de Sergipe, Brasil \\ E-mail: thayane_siqueira@live.com \\ Ariel Oliveira Celestino \\ ORCID: https://orcid.org/0000-0003-3907-187X \\ Universidade Federal de Sergipe, Brasil \\ E-mail: biomedica.ariel@gmail.com \\ Alexrangel Henrique Cruz Santos \\ ORCID: https://orcid.org/0000-0002-3667-5275 \\ Universidade Federal de Sergipe, Brasil \\ E-mail: alex_rangel2020@ hotmail.com \\ Mariana do Rosário Souza \\ ORCID:https://orcid.org/0000-0002-3139-8483 \\ Universidade Federal de Sergipe, Brasil \\ E-mail: enf.mariana.rosario@gmail.com \\ Amanda Francielle Santos \\ ORCID: https://orcid.org/0000-0001-7694-4604 \\ Universidade Federal de Sergipe, Brasil \\ E-mail: francyelly_amanda@hotmail.com \\ Luciene Barbosa \\ ORCID: https://orcid.org/0000-0003-2026-994X \\ Universidade Federal de Sergipe, Brasil \\ E-mail: lucienebarb@bol.com \\ José Rodrigo Santos Silva \\ ORCID: https://orcid.org/0000-0002-1918-7122 \\ Universidade Federal de Sergipe, Brasil \\ E-mail: rodrigo.ufs@gmail.com
}

\begin{abstract}
Resumo
O este estudo visa analisar a tendência-temporal da hanseníase em Sergipe e sua correlação com os determinantes sociais da saúde. Trata-se de um estudo epidemiológico, do tipo ecológico de série temporal, com abordagens descritivas. No estudo serão utilizados os dados do Sistema Nacional de Agravos de Notificação (SINAN) do Ministério da Saúde, relativos ao período de 2007 a 2017.Foram selecionados trêsindicadores epidemiológicos: os coeficientes de casos novos de hanseníase na população geral, em menores de 15 anos e o os casos novos com grau II de incapacidade física, esses foram sujeitos a regressão polinomial. As variáveis independentes foram distribuídas em trêscategorias: Categoria socioeconômica; demográfica e de assistência à saúde, tais variáveis foram correlacionadas estatisticamente através da análise de correlação de Spearman (Significância de 5\%). Para variáveis qualitativas procedeu-se com a categorização dos dados e obtenção das respectivas frequências e percentuais. Em relação à análise descritiva da hanseníase ocorreu um maior predomínio dos homens (51\%), indivíduos com ensino médio incompleto $(52,63 \%)$, morando na zona urbana $(74,36 \%)$, pertencentes a raça indígena, negra e parda $(78 \%)$. Em relação as características clínicas $(50 \%)$ dos indivíduos eram multibacilares. Observou-se uma tendência decrescente para os coeficientes de casos novos de hanseníase na população geral (APC -5,54 e $p<0,000$ ) e em menores de 15 anos (APC $-5,57$ e $p<0,004)$. Em relação aos indicadores socioeconômicos, a razão de renda, a renda per capita, o esgotamento sanitário e o índice de Gini apresentaram uma correlação positiva com os três indicadores de monitoramento da hanseníase.
\end{abstract}

Palavras-chave: Epidemiologia; Saúde pública; Hanseníase; Doenças negligenciadas. 


\begin{abstract}
This study aims to analyze the temporal-trend of care in Sergipe and its correlation with the social determinants of health. It is an epidemiological study, of an ecological type of temporal series, with descriptive approaches. It is not possible to use the data from the National System of Notification of Aggravates (SINAN) of the Ministry of Health, relative to the period from 2007 to 2017. Three epidemiological indicators were selected from the list: the coefficients of new cases of hansenia in the general population, those under 15 years of the new cases with grade II of physical incapacity, these are subject to polynomial regression. Thus, several independent individuals are distributed in three categories: Socioeconomic category; demographic and health care, there are several statistically correlated were through Spearman's correlation analysis (5\% significance). For various qualitative procedures, two data are categorized and the respective frequencies and percentages obtained. In relation to the descriptive analysis of the hansenies or the higher prevalence of two men's $(51 \%)$, individuals with incomplete medium $(52.63 \% \neg)$, living in urban areas (74.36\%), belonging to indigenous, black and brown races (78\%). In relation to the clinical characteristics (50\%), two individuals were multibacillary. Observe a decreasing trend for the coefficients of new cases of hansenia in the general population (APC -5.54 and $p<0.000$ ) and in those under 15 years (APC -5.57 and $p<0.004$ ). In relation to the socio-economic indicators, based on income, income per capita, or the health sector and or the Gini index, they show a positive correlation with the three indicators of monitoring.
\end{abstract}

Keywords: Epidemiology; Public health; Leprosy; Neglected diseases.

\title{
Resumen
}

Este estudio tiene como objetivo analizar la tendencia temporal de la lepra en Sergipe y su correlación con los determinantes sociales de la salud. Se trata de un estudio epidemiológico, de tipo ecológico de series temporales, con enfoques descriptivos. El estudio utilizará datos del Sistema Nacional de Enfermedades Notificables (SINAN) del Ministerio de Salud, para el período 2007 a 2017. Se seleccionaron tres indicadores epidemiológicos: los coeficientes de nuevos casos de lepra en la población general, en menores. 15 años y casos nuevos con discapacidad física grado II, se sometieron a regresión polinomial. Las variables independientes se dividieron en tres categorías: categoría socioeconómica; demográficas y de salud, estas variables se correlacionaron estadísticamente a través del análisis de correlación de Spearman (5\% de significancia). Para las variables cualitativas se categorizaron los datos y se obtuvieron las respectivas frecuencias y porcentajes. En cuanto al análisis descriptivo de la lepra, hubo una mayor prevalencia de hombres $(51 \%)$, individuos con bachillerato incompleto $(52,63 \% \neg)$, residentes en zona urbana $(74,36 \%)$, pertenecientes a la raza indígena, negros y pardos (78\%). En cuanto a las características clínicas (50\%) de los individuos, fueron multibacilares. Se observó una tendencia decreciente para los coeficientes de nuevos casos de lepra en la población general (APC -5.54 yp <0.000) y en menores de 15 años (APC -5.57 yp <0.004). En cuanto a los indicadores socioeconómicos, la relación ingreso, ingreso per cápita, saneamiento y el índice de Gini mostraron una correlación positiva con los tres indicadores de seguimiento de la lepra.

Palabras clave: Epidemiología; Salud pública; Lepra; Enfermedades desatendidas.

\section{Introdução}

A hanseníase é uma doença tropical negligenciada, que ocorre prioritariamente em populações com alta vulnerabilidade social. É causada pelo Mycobacterium leprae ou bacilo de Hansen, assim denominado em homenagem ao médico norueguês Gerhard Henrick Armauer Hansen, que o identificou em 1873. Sua característica infere uma alta infectividade (capacidade de um agente penetrar no organismo humano e desenvolver-se) e baixa patogenicidade (capacidade que o agente possui de gerar sinais e sintomas da doença). Sendo responsável por manifestações dermatoneurológicas e comprometimento da função neural (Lastória et al., 2014; Brasil, 2016 A).

No Brasil, a hanseníase é considerada uma doença de notificação compulsória, seus aspectos epidemiológicos alinhados ao histórico de políticas públicas falhas, que visavam erradicar a doença, fornecem um dos principais fatores para tratá-la como um problema relevante (Brito et al., 2015). Essa problemática está explícita com os dados das estatísticas mundiais, segundo a Organização Mundial da Saúde, em 2016, foram notificados 25.218 casos novos de hanseníase no Brasil, o que representa uma taxa de detecção considerada alta (12,2/100 mil habitantes) (Brasil, 2018 B). 
Dentre as regiões brasileiras, o Nordeste é a terceira região com maior coeficiente de detecção geral (23,8/100 mil habitantes), ficando atrás apenas das regiões Centro-Oeste e Norte respectivamente (Brito et al., 2016). Em Sergipe, o último boletim epidemiológico realizado de 2012 a 2016, demonstrou uma taxa de 17,59 casos novos por 100.000 habitantes, expressando assim, níveis altos de incidência para o estado (Brasil, 2018 B).

Esses números refletem que apesar dos esforços das instituições internacionais e do Ministério da Saúde para a eliminação da hanseníase mediante estratégias e ações programáticas, a doença continua presente no território brasileiro, isso pode ser justificado pela sua distribuição heterogenia no país, com a concentração de casos novos nas regiões com maiores iniquidades sociais (Basso \& Silva, 2017).

A hanseníase não se enquadra em um fenômeno puramente biológico, ao contrário, o adoecimento está ligado aos micro e macrodeterminantes da saúde. Com conhecimento dessas condições sociais e de como eles impactam no surgimento de novos casos, é possível desenvolver estratégias ampliadas que contribuam para uma redução real da sua carga no estado de Sergipe (Schreuder; Noto; Richardus, 2016).

Considerando as informações supracitadas, este estudo visa analisar a tendência-temporal da hanseníase em Sergipe e sua correlação com os determinantes sociais, com o intuito fomentar dados relacionados ao território e suas iniquidades sociais como forma de contribuição para a continuidade da transmissão ativa da doença. Visto que, políticas públicas voltadas para as regiões com maiores desigualdades sociais podem contribuir para a redução dos casos de hanseníase.

\section{Métodos}

\subsection{Desenho do estudo}

Trata-se de um estudo de natureza epidemiológica, do tipo ecológico de série temporal e espacial, com abordagens descritivas. Com o intuito de correlacionar os determinantes sociais da saúde com os casos de hanseníase distribuídos pelo estado de Sergipe, assim como, a tendência temporal dos principais indicadores epidemiológicos da doença. No estudo serão utilizados os dados do Sistema Nacional de Agravos de Notificação (SINAN) do Ministério da Saúde, relativos ao período de 2007 a 2017, disponibilizadas pela Secretaria Estadual de Saúde de Sergipe. Essas fichas contêm informações sociodemográficas e clínicas preenchidas por profissionais da saúde a respeito dos casos de hanseníase notificados (Santos et al., 2019).

As informações sobre a população residente estratificada por faixas etárias serão obtidas por meio do Censo Demográfico de 2010 e de estimativas populacionais, realizados pelo Instituto Brasileiro de Geografia e Estatística (IBGE). Conforme recomendação do Ministério da Saúde, optou-se por apresentar os coeficientes de deteç̧ão por 100.000 habitantes na perspectiva de facilitar a comparação dos coeficientes da hanseníase com outros eventos.

O estudo foi realizado no estado de Sergipe (Figura 1). Sergipe é um dos nove estados localizados na região Nordeste do Brasil cuja capital é Aracaju. Menor estado brasileiro com território de 21.962,10 km2, equivalente a 0,26\% do território nacional, e população estimada de 2.288.116, no ano de 2017. Em sua organização política administrativa, possui 75 municípios agrupados em três mesorregiões e 13 microregiões (IBGE, 2019) 
Figura 1. A) Mapa do Brasil com destaque para a região Nordeste. B) Mapa do estado de Sergipe com as divisões municipais.

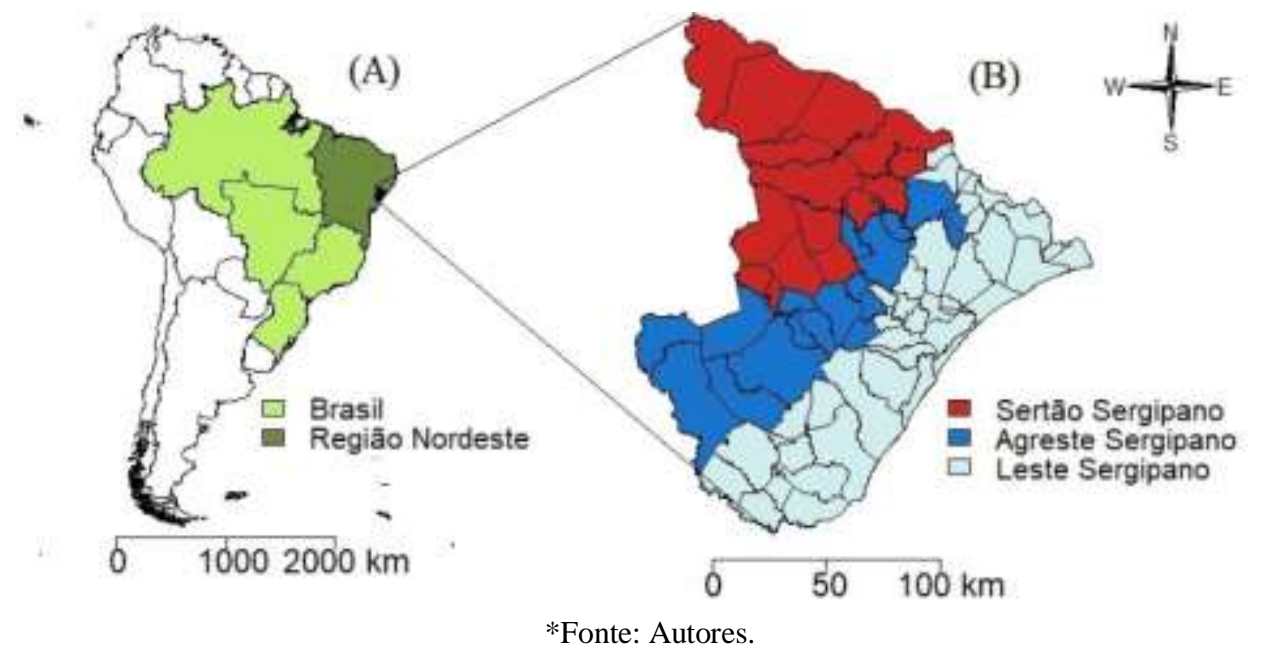

Para a análise dos indicadores epidemiológicos, os mesmos foram descritos com as taxas de incidência anuais, a partir do cálculo do número de casos novos da hanseníase dividido pelo número de residentes no mesmo local no período considerado, multiplicado pela constante 100.000. Os dados foram obtidos diretamente do Sistema de Informação de Agravos de Notificação (SINAN) e no Instituto Brasileiro de Geografia e Estatística (IBGE) para o do estado de Sergipe, Brasil (IBGE, 2010).

Os indicadores epidemiológicos selecionados foram: Taxa de incidência de hanseníase por 100 mil habitantes; Taxa de incidência de hanseníase em menores de 15 anos, por 100 mil habitantes ( $<15$ anos de idade); Taxa de casos novos com grau 2 de incapacidade, por 100 mil habitantes (taxa de detecção) Proporção de casos de hanseníase, segundo gênero entre o total de casos novos;Proporção de casos segundo classificação operacional entre o total de casos novos. Todos calculados com base $\mathrm{n}$ nas "Diretrizes para vigilância, cuidado e eliminação da hanseníase como um problema de saúde pública ". Para o percentual de cura, o numerador foi o número de casos novos de hanseníase, residentes em local específico, diagnosticados nos anos das coortes e curados até 31/12 do ano da avaliação (Brasil, 2017 C).

As variáveis independentes foram escolhidas com base nos estudos realizados sobre os determinantes sociais da saúde e hanseníase, e de acordo com as características demográficas e socioeconômicas dos municípios (Assis, et al., 2018; Monteiro, et al., 2017; Rangel, 2016). A partir disso as variáveis independentes foram divididas em três categorias: Categoria socioeconômica: Índice de Desenvolvimento humano (IDH), razão de renda, índice de Gini, taxa de analfabetismo, taxa de pessoas com baixa renda, renda média domicílio per capita, taxa de desemprego, PIB- per capita, saneamento inadequado. Categoria Demográfica: Taxa de urbanização, densidade demográfica. Categoria de Assistência à saúde: Taxa de mortalidade infantil, cobertura da ESF.

\subsection{Análise Estatística}

Foi empregada a regressão polinomial para o cálculo da variação anual dos indicadores, no período entre 2007 e 2017. Esse método de análise consiste em uma regressão linear segmentada, com uso de variáveis dummy, para identificar pontos em que há modificação da tendência e estimar a Variação Percentual Anual (APC), e a Média da Variação Percentual Anual (AAPC) considerando o período inteiro da série com intervalo de confiança de 95\% (IC 95\%. A tendência foi classificada em crescente, decrescente e estacionária (Monteiro et al., 2015).

Para a associação entre as variáveis dependentes e independentes, buscou-se avaliar a existência de correlação estatística entre os indicadores epidemiológicos e as categorias socioeconômica, demográfica e de assistência à saúde 
utilizando a análise de correlação de Spearman (Significância de 5\%). O coeficiente de correlação varia entre -1 e +1 e indica o grau de associação linear entre os indicadores e a detecção de hanseníase. Valores próximos à unidade indicam forte correlação, quando positivos esta é direta e quando negativo é indireta, isto é, inversamente proporcional. O coeficiente de Spearman torna-se menos sensível a valores extremos do que o coeficiente de Pearson, os dados em questão apresentam grande variabilidade de valores entre os municípios, devido à heterogeneidade da endemia e desigualdades socioeconômica (Assis et al., 2018; Gracie et al.,2017).

Para variáveis qualitativas a análise descritiva procedeu-se com a categorização dos dados e obtenção das respectivas frequências e percentuais. Já as variáveis quantitativas foram calculadas as medidas de tendência central (média), variabilidade (desvio padrão) e posição (mínimo e máximo).

\section{Resultados}

Dos 4590 casos novos analisados no período de 2007 a 2017, observou dentre eles, que 2328 (51\%) eram do sexo masculino, enquanto 2242 (49\%) eram do sexo feminino. A idade dos pacientes variou de 1 a 100 anos, com a média da idade de 43 anos, (desvio padrão de \pm 18,9). A faixa etária adulto/jovem foi a mais acometida 1560 (33, 99\%), seguida da população adulta $1175(25,60 \%)$. Os menores de 15 anos corresponderam 319 (6,95\%) dos casos. Os negros, indígenas e pardos, representaram a maioria dos portadores da doença 3441(78\%), já os considerados brancos ou amarelo obtiveram um menor percentual 932 (22\%) (Tabela 1).

A partir das análises foi possível perceber o nível de escolaridade baixa entre os hansêmicos, constituindo-se de 473 $(10,24 \%)$ de analfabetos e 2409 (52,63\%) com o ensino fundamental completo ou incompleto. Grande parte residia em áreas urbanas 2400 (74,76\%), ao mesmo tempo que, os moradores de áreas rurais totalizaram cerca de 795(17,16\%) (Tabela 1).

Tabela 1 - Distribuição das características sociodemográficas da hanseníase no estado de Sergipe, no período de 2007-2017.

\begin{tabular}{|c|c|c|}
\hline Variáveis & $\mathrm{n}$ & $\%$ \\
\hline \multicolumn{3}{|l|}{ Sexo } \\
\hline Feminino & 2242 & $49 \%$ \\
\hline Masculino & 2328 & $51 \%$ \\
\hline \multicolumn{3}{|l|}{ Escolaridade } \\
\hline Analfabeto & 473 & $10,24 \%$ \\
\hline E. Fundamental & 2409 & $52,63 \%$ \\
\hline E. Médio & 709 & $15,42 \%$ \\
\hline Superior & 207 & $4,3 \%$ \\
\hline \multicolumn{3}{|l|}{ Zona } \\
\hline Urbana & 3400 & $74,36 \%$ \\
\hline Periurbana & 61 & $1,45 \%$ \\
\hline Rural & 795 & $17,16 \%$ \\
\hline \multicolumn{3}{|l|}{ Raça } \\
\hline Branco/Amarelo & 932 & $22 \%$ \\
\hline Negro/indígena/pardo & 3441 & $78 \%$ \\
\hline \multicolumn{3}{|l|}{ Idade } \\
\hline$<15$ & 319 & $6,95 \%$ \\
\hline $15-24$ & 518 & $11,29 \%$ \\
\hline $25-44$ & 1560 & $33,99 \%$ \\
\hline 45-59 & 1175 & $25,60 \%$ \\
\hline$>60$ & 1018 & $22,18 \%$ \\
\hline
\end{tabular}


Em relação as características clínicas da hanseníase no período estudado podemos inferir uma paridade em relação a classificação operacional dos portadores da endemia 2293 (50\%) paucibacilares e 2297 (50\%) multibacilares. As formas clínicas da doença também demostraram uma igualdade em sua distribuição com uma maior prevalência das formas tuberculóide 1661(24,42\%) e da virchowina 1009 (22,84\%), a mais severa dentre as outras (Tabela 2).

A baciloscopia do raspado intradérmico é utilizada como um auxiliador no diagnóstico diferencial com outras doenças dermatoneurológicas. Foi realizada em 2766 pessoas, sendo positivas 1188 (26\%) e 1578 (34,3) negativas. Em relação ao esquema terapêutico poliquimioterápico -PQT $2282(49,7 \%)$ faziam uso do tratamento direcionado para paucibacilares 6 meses, enquanto os que faziam uso do tratamento multibacilar 12 meses representaram um total de 2076 (45,3\%), outros esquemas terapêuticos correspondiam a 232 (5\%) dos casos (Tabela 2).

Em relação ao modo de deteç̧ão da hanseníase, os indivíduos detectados por meio do padrão de encaminhamento caracterizaram-se como a maioria 2215 (48,20\%), já os descobertos por meio da demanda espontânea totalizaram 1951 $(42,50)$. Os detectados por meio do exame dos contatos integraram 137(3\%). Quanto ao grau de incapacidade, salienta-se que, em sua subdivisão, o grau 0 correspondeu a 2463 (53,3\%) dos casos, o grau I 393 (9\%), e por fim o grau II 132 (3\%), sendo este último o mais incapacitante (Tabela 2).

Tabela 2 - Distribuição das características clínicas dos casos novo da hanseníase no estado de Sergipe, no período de 20072017.

\begin{tabular}{|c|c|c|}
\hline Classificação Operacional & $\mathrm{n}$ & $\%$ \\
\hline Paucibacilar & 2293 & $50 \%$ \\
\hline Multibacilar & 2297 & $50 \%$ \\
\hline \multicolumn{3}{|l|}{ Formas clínicas } \\
\hline Indeterminada & 1090 & $22,32 \%$ \\
\hline Tuberculóide & 1661 & $24,42 \%$ \\
\hline Dimorfa & 978 & $22,57 \%$ \\
\hline Virchowiana & 1009 & $22,84 \%$ \\
\hline \multicolumn{3}{|l|}{ Baciloscopia } \\
\hline Positiva & 1188 & $26 \%$ \\
\hline Negativa & 1578 & $34,3 \%$ \\
\hline Não realizada & 1824 & $39,7 \%$ \\
\hline \multicolumn{3}{|l|}{ Esquema Terapêutico } \\
\hline Paucibacilar 6 doses & 2282 & $49,7 \%$ \\
\hline Multibacilar 12 doses & 2076 & $45,3 \%$ \\
\hline Outros esquemas & 232 & $5 \%$ \\
\hline \multicolumn{3}{|l|}{ Modo de Detecção } \\
\hline Demanda espontânea & 1951 & $42,50 \%$ \\
\hline Encaminhamento & 2215 & $48,20 \%$ \\
\hline Exame de coletividade & 82 & $2 \%$ \\
\hline Exame de contatos & 137 & $3 \%$ \\
\hline Outros modos & 145 & $4,3 \%$ \\
\hline \multicolumn{3}{|l|}{ Grau de Incapacidade } \\
\hline Grau 0 & 2463 & $53,3 \%$ \\
\hline Grau 1 & 393 & $9 \%$ \\
\hline Grau 2 & 132 & $3 \%$ \\
\hline Não avaliados & 478 & $10 \%$ \\
\hline
\end{tabular}

Fonte: Dados da pesquisa.

Observou-se que a maior taxa de incidência ocorreu no ano de 2007 (26, 5/100.000hab), classificada como muito alta segundo parâmetros do Ministério da Saúde, enquanto a menor taxa foi registrada no ano de 2016 (12,8/100.000hab), 
considerada alta segundo parâmetros nacionais .Os resultados para o coeficiente de detecção geral demonstraram uma tendência decrescente em todo o período 2007-2017 (APC -5,54 e p<0,000) (Tabela 3).

O coeficiente de deteç̧ão da hanseníase em menores de 15 anos registrou a maior incidência no ano de 2007 (7, 4/100.000hab), nesse ano a endemia foi considerada muito alta, todavia apresentou as menores taxas nos anos de 2015 e 2016 (3,6/100.000hab) em ambos os anos. Semelhante a taxa de detecção geral, o coeficiente de detecção em menores de 15 anos apresentou uma tendência decrescente no período de 2007-2017 (APC -5,57 e $p<0,004$ ) (Tabela 3).

A análise do coeficiente de casos novos com grau II de incapacidade mostrou oscilação durante o período, apresentado uma taxa de (1,13/100.000hab) em 2007, (2, 6/100.000hab) em 2012, ambas consideradas muito altas. A menor taxa foi registrada em 2016 (0,79/100.000hab), considerada baixa. Foi observado duas tendências, sendo a primeira de crescimento entre 2007 e 2012 (APC 13,14 e $p<0,047$ ) e segunda decrescente entre 2012-2016 (APC -25,16 e $p<0,028$ ). (Tabela 3).

A proporção de indivíduos do sexo feminino obteve o maior percentual de casos em 2011 (53,5\%) e a menor em 2016 $(43,8)$ (Tabela 3). Vale ressaltar que ainda não existem parâmetros definidos pelo Ministério da Saúde para a mensuração desse indicador. No que tange a tendência podemos salientar que a mesma se manteve estacionária durante todo o período de 2007 a 2017 ( $p>0,191)$. A proporção de casos multibacilares apresentou sua maior porcentagem no ano de 2007 (60\%), enquanto a menor foi representada pelo percentual de $(44,7 \%)$ em 2007. A partir da regressão segmentada observou-se uma tendência crescente de $2007-2017$ (APC 2,12 e $p<0,004$ ). (Tabela 3).

Tabela 3 - Análise Temporal dos indicadores epidemiológicos de monitoramento do processo de eliminação da hanseníase. Sergipe- Brasil- 2007-2017.

\begin{tabular}{|c|c|c|c|c|c|c|}
\hline Indicador & APC (IC95\%) & Tendência & $P$-valor & APC (IC95\%) & Tendência & P-valor \\
\hline $\begin{array}{l}\text { Coeficiente de } \\
\text { detecção de } \\
\text { casos novos na } \\
\text { população } \\
\text { geral }\end{array}$ & $\begin{array}{c}-5,54 \\
{[-7.80 ;-3.29]} \\
2007-2017\end{array}$ & Decrescente & 0,000 & - & - & - \\
\hline $\begin{array}{c}\text { Coeficiente de } \\
\text { detecção em } \\
<15 \text { anos/100 } \\
\text { mil }\end{array}$ & $\begin{array}{c}-5,57 \\
{[-8.65 ;-2.25]} \\
2007-2017\end{array}$ & Decrescente & 0,004 & - & - & - \\
\hline $\begin{array}{c}\text { Coeficiente de } \\
\text { casos novos } \\
\text { com grau } \\
\text { II/100 mil }\end{array}$ & $\begin{array}{c}13,14 \\
{[2.66 ; 26.01]} \\
2007-2012\end{array}$ & Crescente & 0,047 & $\begin{array}{c}-25,16 \\
{[-45.25 ;-5.07]} \\
2012-2016\end{array}$ & Decrescente & 0,028 \\
\hline $\begin{array}{l}\text { \% de casos } \\
\text { gênero } \\
\text { feminino }\end{array}$ & $\begin{array}{c}- \\
2007-2017\end{array}$ & Estacionária & 0,191 & - & - & - \\
\hline $\begin{array}{c}\% \text { Casos } \\
\text { multibacilares }\end{array}$ & $\begin{array}{c}2,12 \\
{[0.90 ; 3.34]} \\
2007-2017\end{array}$ & Crescente & 0,004 & - & - & - \\
\hline
\end{tabular}

Fonte: Dados da pesquisa.

$\mathrm{Na}$ Tabela 4 podemos observar a correlação de Spearman entre os indicadores epidemiológicos da hanseníase (Coeficiente de deteç̧ão geral de casos novos, Coeficiente de detecção em menores de 15 anos, coeficiente de grau II de incapacidade) e os indicadores socioeconômicos, demográficos e da saúde. Em relação aos indicadores socioeconômicos descritos na Tabela 1, a razão de renda, a renda per capita, o esgotamento sanitário e o índice de Gini apresentaram uma 
correlação positiva com os três indicadores de monitoramento da hanseníase. Podemos inferir então que, quanto maior a desigualdade relacionada a distribuição de renda, e maior a cobertura do esgotamento sanitário, consequentemente haverá um aumento na detecção dos casos de hanseníase para os três indicadores. (Tabela 4).

O IDH apresentou correlação positiva com o coeficiente de detecção em menores de 15 anos e com o coeficiente de grau II de incapacidade física, sendo a correlação entres eles considerada fraca $(r=0,14)$ e $(r=0,17)$, respectivamente. Já a taxa de pessoas com baixa renda correlacionou-se inversamente com os três indicadores, sugerindo que, quanto menor a taxa de pessoas com baixa renda, maior o coeficiente de detecção dos indicadores da hanseníase. A taxa de analfabetismo apresentou correlação inversa com os o coeficiente de deteç̧ão em menores de 15 anos e com o coeficiente de grau II de incapacidade física (Tabela 4).

Quantos aos indicadores sociodemográficos, a taxa de urbanização e densidade demográfica também apresentaram correlação positiva com os coeficientes epidemiológicos da hanseníase, sugerindo que os municípios com maior urbanização e densidade demográfica apresentam a maior taxa de detecção para os indicadores supracitados. E por último os indicadores de saúde, a mortalidade infantil apresentou correlação positiva apenas com o coeficiente de detecção em menores de 15 anos, enquanto a cobertura da ESF apresentou correlação positiva com o coeficiente de detecção geral e correlação inversa com o coeficiente de detecção em menores de 15 anos e com o coeficiente de grau II de incapacidade física (Tabela 4).

Tabela 4 - Correlação estatisticamente significativa entre as variáveis independentes e os indicadores epidemiológicos da hanseníase em Sergipe-Brasil, 2007-2017.

\begin{tabular}{|c|c|c|c|c|c|c|}
\hline Indicadores & $\begin{array}{c}\text { Coef. de } \\
\text { Detecão } \\
\text { Geral }\end{array}$ & P-Valor & $\begin{array}{c}\text { Coef. } \\
\text { detecção em } \\
<15 \text { anos }\end{array}$ & $P$-valor & $\begin{array}{c}\text { Coeficiente } \\
\text { de grau II de } \\
\text { incapacidade } \\
\text { física }\end{array}$ & P-Valor \\
\hline $\begin{array}{c}\text { Razão de } \\
\text { Renda }\end{array}$ & $0,13^{*}$ & 0,000 & $0,09^{*}$ & 0,015 & $0,008^{*}$ & 0,024 \\
\hline IDH & 0,04 & 0,309 & $0,14^{*}$ & 0,000 & $0,17 *$ & 0,000 \\
\hline $\begin{array}{c}\text { Taxa de } \\
\text { analfabetismo }\end{array}$ & 0,01 & 0,781 & $-0,18^{*}$ & 0.000 & $-0,13^{*}$ & 0.000 \\
\hline Índice de Gini & $0,16^{*}$ & 0,000 & $0,11^{*}$ & 0,001 & $0,008 *$ & 0,024 \\
\hline $\begin{array}{c}\text { Taxa de } \\
\text { pessoas com } \\
\text { baixa renda }\end{array}$ & $-0,09 *$ & 0,007 & $-0,19^{*}$ & 0,000 & $-0,22 *$ & 0,000 \\
\hline $\begin{array}{c}\text { Renda per } \\
\text { Capita }\end{array}$ & $0,10^{*}$ & 0,003 & $0,23 *$ & 0,000 & $0,20^{*}$ & 0,000 \\
\hline $\begin{array}{c}\text { Taxa de } \\
\text { desemprego }\end{array}$ & 0,01 & 0,880 & - & - & 0,04 & 0,294 \\
\hline $\begin{array}{l}\text { Esgotamento } \\
\text { sanitário }\end{array}$ & $0,26^{*}$ & 0,000 & $0,21^{*}$ & 0,000 & $0,20^{*}$ & 0,000 \\
\hline $\begin{array}{c}\text { Taxa de } \\
\text { urbanização }\end{array}$ & $0,06^{*}$ & 0,073 & $0,18^{*}$ & 0,000 & $0,11^{*}$ & 0,002 \\
\hline $\begin{array}{c}\text { Densidade } \\
\text { demográfica }\end{array}$ & $0,08^{*}$ & 0,016 & $0,26^{*}$ & 0,000 & $0,15^{*}$ & 0,000 \\
\hline $\begin{array}{c}\text { Cobertura } \\
\text { ESF }\end{array}$ & $0,19^{*}$ & 0,000 & $-0,22 *$ & 0,000 & $-0,18^{*}$ & 0,000 \\
\hline $\begin{array}{l}\text { Mortalidade } \\
\text { infantil }\end{array}$ & 0,03 & 0,436 & $0,07 *$ & 0,044 & 0,00 & 0,898 \\
\hline
\end{tabular}




\section{Discussão}

Em Sergipe ocorreu uma maior prevalência de casos de hanseníase no sexo masculino em relação ao feminino, segundo Sarmento et al., (2015), a literatura apresenta divergência em relação à prevalência da hanseníase quanto ao sexo, visto que, apesar dos homens apresentarem um maior contato social em ambientes urbanos, onde bacilo transita com maior facilidade, os mesmos não buscam os serviços de saúde o que seria contributivo para uma diminuição do diagnóstico precoce nesse grupo. Assim, alguns estudos da hanseníase no Brasil tenderiam para uma maior prevalência do sexo feminino. Vale salientar que o baixo nível de autocuidado e o menor acessam a informações são pontos importantes que enfatizam o gênero como determinante relevante para a manutenção da doença (Souza et al.,2018).

Com relação à raça/cor ocorreu um predomínio da hanseníase entre os negros pardos e indígenas, segundo Souza et al., (2019), essa maior representatividade ligada a esses grupos remete a um processo histórico marcado por desigualdades sociais, individuais ou programáticas. As doenças negligenciadas, como a hanseníase endossam a narrativa histórica da colonização, miscigenação, movimentos migratórios, organização e espacial. A população adulta foi a mais acometida pela hanseníase em Sergipe, esses dados são condizentes com literatura, segundo Basso e Silva. (2017) a hanseníase é predominante na faixa economicamente ativa, ressalta-se que doenças que apresentam longos períodos de incubação e dificuldades para o diagnóstico precoce e se manifestam tardiamente, podendo incapacitar os indivíduos levando a interrupção das atividades laborais.

Com relação à escolaridade, a maior parte dos hansêmicos apresentaram baixa escolaridade, esse dado é bastante citado nos estudos epidemiológicos voltados para hanseníase, uma vez que o nível educacional está relacionado com a falta de entendimento sobre a doença, demora para buscar os serviços de saúde, diagnóstico precoce e adesão ao tratamento (Silva et al., 2105). Costa el al., (2019) infere que o nível educacional elevado é um fator protetor para a ocorrência de incapacidades físicas, já que, o conhecimento individual pode gerar um efeito positivo na diminuição dos casos graves doença. A maior parte dos portadores da hanseníase residia em áreas urbanas, segundo Schlickman e Guerino. (2012), os centros urbanos oferecem grande parte das atividades laborais o que propicia a aglomeração de pessoas, facilitando a sua disseminação.

Observa-se nesse estudo que metade dos casos pertenciam as formas clínicas (Virchoviana e Dimorfa), responsáveis pela transmissão ativa da doença, e que podem levar ao grau incapacitante. Os multibacilares é grupo mais fragilizado, tendo em vista os desdobramentos de natureza econômica, social e psicológica ocasionados pela evolução dessa patologia. É necessário enfatizar a importância do diagnóstico precoce e da completude do tratamento para a cura da doença, entretanto os determinantes sociais da saúde influenciam diretamente no desfecho dos indivíduos portadores de hanseníase (Carlos et al.,2019).

Em Sergipe ocorreu uma diminuição na taxa de incidência de hanseníase na população geral; taxa de incidência de hanseníase em menores de 15 anos, ( $<15$ anos de idade) e na taxa de casos novos com grau 2 de incapacidade no período de 2007 a 2017. Segundo Ribeiro et al. (2018), a hanseníase vem diminuindo a nível mundial, entretanto sua eliminação ainda é uma proposta desafiadora, tendo em vista as disparidades regionais que contribuem para manutenção da doença. Ribeiro et al. (2018), ainda ratifica que ocorreu um aumento na prevalência da hanseníase entre os anos de 2006 e 2009, fruto do empenho do Ministério da Saúde em detectar a doença e erradica-la. Essa narrativa corrobora com esse estudo, visto que, as taxas de detecção no ano de 2007 foram altas para os três indicadores supracitados. Na série histórica estudada destaca-se a tendência crescente dos casos multibacilares, em um estudo realizado por Souza et al. (2018) na Bahia, no período de 2001 a 2014 , ocorreu um aumento de $62 \%$ para os casos multibacilares. A autora supracitada endossa a ideia já discutida por outros pesquisadores, que a falta de capacitação dos profissionais de saúde para diagnosticar precocemente a doença, atrelada ao estigma e o preconceito, favorecem o silêncio em torno do debate da hanseníase. 
A hanseníase não pode ser vista apenas no âmbito biológico, ela não ocorre de forma linear seguindo a linha causa e efeito, a mesma é condicionada por diversos aspectos sejam eles de ordem física, socioeconômica, cultural e psíquica. Sob esse ponto de vista a determinação social traz reflexões a respeito da equidade e integralidade a saúde, princípios presentes desde a reforma sanitária. É necessária a intersecção entre o processo saúde doença e as iniquidades em saúde (Lopes e Rangel 2014).

O estudo veio explicitar por meio dos resultados que quanto maior a desigualdade em relação à distribuição de renda, haverá um aumento da deteç̧ão dos casos de hanseníase, esse dado é compatível com outros estudos já realizados como o de Souza et al. (2019), que analisou a correlação dos determinantes sociais da saúde com os indicadores epidemiológicos da hanseníase no período de 2001 a 2015, nesse estudo foi inferido que que municípios com os maiores IDH apresentavam também maiores índices de desigualdade de renda. Um outro estudo realizado por Freitas (2018), mostrou que os municípios brasileiros com maior desigualdade são aqueles que possuem maior carga da doença. A taxa de urbanização e a densidade populacional apresentou correlação positiva com os coeficientes epidemiológicos da hanseníase. Um estudo realizado por Monteiro et al. (2017), ressalta que o processo endêmico da doença pode associar-se a alta densidade demográfica e ao processo de urbanização acelerado e sem planejamento, esses fatores contribuem para um contato maior entre as pessoas e maior circulação do bacilo. Indivíduos que vivem em regiões mais periféricas das cidades, no geral, vivem em condições inapropriadas de moradia e sofrem com a falta de acesso aos serviços de saúde, assim, esses grupos se encontram em alta situação de vulnerabilidade social, elevando o risco de contrair a doença.

A importância de uma maior cobertura da Estratégia da Saúde da Família (ESF), na detecção dos casos é enfatizada na literatura, principalmente no que tange ao agravamento dos casos, o acompanhamento dos pacientes é de fundamental relevância para melhor compreensão e motivação quanto à credibilidade do diagnóstico precoce, tratamento e cura. Alguns estudos como de Ribeiro et al. (2018). Apontam que a estrutura organizacional do sistema de saúde tenha uma maior influência na situação epidemiológica da hanseníase do que as disparidades socioeconômicas, haja visto que, uma maior cobertura da Estratégia de Saúde da Família (ESF) contribui efetivamente para a prevenção, promoção e tratamento das transmissíveis.

Esse estudo apesar de relevante possui algumas limitações relacionadas aos dados secundários provenientes dos sistemas de informação, que nem sempre representam a realidade do estado como um todo, devido às subnotificações. Outro ponto é a própria natureza do estudo de cunho ecológico, é necessário o cuidado com o viés ecológico ou falácia ecológica, que ocorre quando uma associação observada a um nível agregado é transportada para um nível individual.

\section{Conclusão}

Em síntese, o estudo revela que apesar de ter ocorrido uma diminuição na detecção da hanseníase nos principais indicadores epidemiológicos (Coeficiente de detecção geral, coeficiente de detecção nos menores de 15 anos e casos novos com grau II de incapacidade física), a doença ainda persiste no estado, e sua erradicação é um projeto a longo prazo, tendo em vista a sua natureza multifacetada atrelada a fat6res sociais e econômicos. Em Sergipe, é notório pela própria caracterização do perfil da doença nos indivíduos a sua ligação com as iniquidades sociais.

Os gestores responsáveis pela programação da saúde devem se atentar aos grupos de risco: homens, analfabetos ou com ensino fundamental incompleto, na faixa etária economicamente ativa, pertencentes a raça negra, parda ou indígena. Além disso, Os indivíduos multibacilares com as formas mais graves da doença (dimorfa e virchowiana) são os responsáveis pela transmissão ativa da hanseníase. Ressalta-se então, a necessidade de um acompanhamento mais estruturado desses pacientes visando a integralidade e a concretização do vínculo enquanto princípio primordial da Atenção Básica.

O conhecimento sobre os determinantes sociais da saúde contribui para o desenvolvimento de estratégias que visem a erradicação dessa doença milenar. Investimentos direcionados na melhoria das condições de vida da população, assim como, 
maior cobertura da ESF podem ser medidas efetivas para melhorar a detecção dos casos. Desse modo, esse estudo visa fomentar outras pesquisas que relacionem os DSS a doenças tropicais negligenciadas como a hanseníase.

\section{Referências}

Assis, I. S., et al. (2018). Social determinants, their relationship with leprosy risk and temporal trends in a triborder region in Latin America. PLOS Neglected Tropical Diseases. 12.(4).1-17. https://doi.org/10.1371/journal.pntd.0006407

Basso, M. E. M., \& Silva, R. L. F. (2017). Perfil clínico-epidemiológico de pacientes acometidos pela hanseníase atendidos em uma unidade de referência. Rev Soc Bras Clin Med. 15(1):27-32. https://pesquisa.bvsalud.org/portal/resource/pt/biblio-833138

Brasil. (2016) A. Ministério da Saúde. Diretrizes para vigilância, atenção e eliminação da hanseníase como problema de saúde pública. Manual Técnico Operacional. Brasília DF- https://portalarquivos2.saude.gov.br/images/pdf/2016/fevereiro/04/diretrizes-eliminacao-hanseniase-4fev16-web.pdf

Brasil.(2017) Ministério da Saúde. Secretaria de Vigilância em Saúde e Departamento de Vigilância e Doenças Transmissíveis.Guia Prático sobre a Hanseníase. Brasília DF- https://portalarquivos2.saude.gov.br/images/pdf/2017/novembro/22/Guia-Pratico-de-Hanseniase-WEB.pdf

Brasil. (2018) C. Ministério da Saúde, Secretaria de Vigilância em Saúde. Boletim Epidemiológico. 49(4). https://portalarquivos2.saude.gov.br/images/pdf/2018/novembro/19/2018-032.pdf

Brito, A. L., Monteiro, L D., Junior, A. N. R., Heukelbach, J., \& Alencar, C. H. (2016). Tendência temporal da hanseníase em uma capital do Nordeste do Brasil: epidemiologia e análise por pontos de inflexão, 2001 a 2012.Revista. Brasileira de Epidemiologia. 19(1), 194-204. https://doi.org/10.1590/19805497201600010017

Brito, K. K. G., Andrade, S. S. C.,Santana, E. M. F., Peixoto, V. B., Nogueira, J. A, \& Soares, M. G. O. (2015). Análise epidemiológica da hanseníase em um estado endêmico do nordeste brasileiro.Rev Gaúcha Enferm. 36, 24-30. https://www.scielo.br/pdf/rgenf/v36nspe/0102-6933-rgenf-36-spe-0024.pdf

Freitas L. R. S., Duarte E. C., \& Garcia L.P.(2014). Leprosy in Brazil and its association with characteristics of municipalities: ecological study, 2009-2011. Trop Med Int Health.19(10):1216-25. https://doi.org/10.1111/tmi.12362

Instituto Brasileiro de Geografia e Estatística (IBGE) (2017). https://cidades.ibge.gov.br/municipio/280390

Lastória, J. C., \& Abreu, M. A. M. M.(2012). Hanseníase: diagnóstico e tratamento.17(4):173-9. http://files.bvs.br/upload/S/1413-9979/2012/v17n4/a3329.pdf

Lastória, J. C., Almeida, T. S. C., Putinatti, M. S. M., \& Padovani, C. R. (2018). Effectiveness of the retreatment of patients with multibacillary leprosy and episodes of erythema nodosum leprosum and/or persistent neuritis: a single-center experience. Anais Brasileiros de Dermatologia. 93 n. ((2). 181-184. http://dx.doi.org/10.1590/abd1806-4841.20185387

Monteiro L. D, Martins-Melo, F. R, Brito, A. L, Alencar, C. H, \& Heukelbah, J.(2015). Padrões espaciais da hanseníase em um estado hiperendêmico no Norte do Brasil, 2001-2012. Rev. Saúde Pública [online]. 49(84):1-8. https://www.scielo.br/pdf/rsp/v49/pt_0034-8910-rsp-S0034-89102015049005866.pdf

Pinheiro, M. G. C et al. (2019). Análise contextual da atenção à saúde na alta em hanseníase: uma revisão integrativa. Revista Gaúcha de Enfermagem, 40, e20180258. https://doi.org/10.33448/rsd-v9i8.4923

Rangel, M. E. S.(2016). Dinâmica espacial e contingências socioambientais da hanseníase no estado do Maranhão: avaliação de riscos e vulnerabilidades em área hiperendêmica. [Tese]. São Paulo: Universidade de São Paulo. https://repositorio.usp.br/item/002791997

Ribeiro, M. D. A., Silva, J. C. A., \& Oliveira, S. B. (2018). Epidemiologic study of leprosy in Brazil: reflections on elimination goals. Rev Panam Salud Publica v42. n07 p-7.https://www.scielosp.org/article/rpsp/2018.v42/e42/

Sarmento, A. P. A., Pereirão, A. M., Ribeiro, F., Castro, J. L., Ameida, M. B., \& Ramos, N. M.(2015). Perfil epidemiológico da hanseníase no período de 2009 a 2013 no município de Montes Claros (MG). Rev Soc Bras Clin Med. 13(3):180-185. http://files.bvs.br/upload/S/1679-1010/2015/v13n3/a5389.pdf

Silva, C. L. M. et al.(2017). Spatial distribution of leprosy in Brazil: a literature review. Rev Soc Bras Med Trop 50(4):439-449. https://www.scielo.br/scielo.php?script=sci_arttext\&pid=S0037-86822017000400439

Schlickman, E. E. D, Guerino, M. R. (2012). Relação entre o número de casos de hanseníase e o número de unidades básicas na cidade de Primavera do LesteMT, Brasil. J Health Sci Inst.30(2):150-5. http://repositorio.unip.br/wp-content/uploads/2020/12/V30_n2_2012_p150-155.pdf

Schreuder P. A., Noto S., \& Richardus J. H. (2016). Epidemiologic trends of leprosy for the 21st century. Clin Dermatol. 34(1):24-31. https://doi.org/10.1016/j.clindermatol.2015.11.001

Souza, E. A., et al. (2018) Leprosy and gender in Brazil: trends in an endemic area of the Northeast region, 2001-2014. Rev Saude Publica, 52:20. 12. http://www.scielo.br/pdf/rsp/v52/0034-8910-rsp-S1518-87872018052000335.pdf

Souza C. D. F., Franca-Rocha, W. J. S. F., \& Lima, R. S. (2014). Distribuição espacial da endemia hansênica em menores de 15 anos em Juazeiro-Bahia, entre 2003 e 2012. Hygeia. 10(19):35-49. http://www.seer.ufu.br/index.php/hygeia/article/view/27120 